\title{
Hyperventilation-induced nystagmus in patients with vestibular schwannoma
}

\author{
Lloyd B. Minor, MD; Thomas Haslwanter, PhD; Dominik Straumann, MD; and David S. Zee, MD
}

\begin{abstract}
Article abstract-Objective: To analyze the nystagmus evoked by hyperventilation in patients with unilateral vestibular schwannoma and to use this information to predict the effects of hyperventilation on individual ampullary nerves. Methods: Three-dimensional scleral search coil eye movement recording techniques were used to record the magnitude and time course of eye movements in six patients with unilateral vestibular schwannoma and hyperventilation-induced nystagmus. The presenting complaints in five of these patients were vertigo or dysequilibrium. Results: The eye movement response to hyperventilation was a "recovery" nystagmus with slow-phase components corresponding to excitation of the affected vestibular nerve. Projection of the eye velocity vector into the plane of the semicircular canals revealed that fibers arising from the ampulla of the horizontal canal were most affected by hyperventilation with lesser activation of fibers to the superior canal and smaller, more variable responses from posterior canal fibers. Conclusions: The three-dimensional characteristics of the nystagmus evoked by hyperventilation in patients with vestibular schwannoma provide insight into the vestibular end organs affected by the tumor and the mechanism responsible for the nystagmus. This finding indicates that hyperventilation resulted in a transient increase in activity from these partially demyelinated axons. Key words: Acoustic neuroma-Recovery nystagmus-Three-dimensional eye movements-Hyperventilation-Vestibular schwannoma.
\end{abstract}

NEUROLOGY 1999;53:2158-2167

Hyperventilation has been reported to increase the spontaneous nystagmus following vestibular neurectomy ${ }^{1}$ and can induce a nystagmus in certain vestibular disorders. Nystagmus has been observed following hyperventilation in patients with acoustic neuroma. ${ }^{2,3}$ Although termed "acoustic," these schwannomas actually arise from the vestibular portion of the eighth cranial nerve. ${ }^{4}$ Hyperventilationinduced nystagmus has also been reported in a patient with an epidermoid tumor eroding the petrous bone, ${ }^{5}$ in perilymph fistula or microvascular compression of the vestibular nerve ${ }^{6}$ and in patients with cerebellar dysfunction. ${ }^{7}$ Dizziness and lightheadedness, often accompanied by numbness and paresthesias, can be elicited by hyperventilation in patients with anxiety disorders. ${ }^{8,9}$

The plane in which the eyes move during the nystagmus evoked by hyperventilation provides information about which semicircular canals are affected. This relationship between eye movements and canal planes was first identified from studies performed in cats and in monkeys. The eyes were noted to move in the plane of the activated canal following electrical stimulation of nerves to each of the ampullae of the semicircular canals. ${ }^{10}$ The eye movements evoked by electrical stimuli that activate afferent nerve fibers innervating all of the vestibular end organs correspond to those predicted based upon a linear summation of the responses from each of the semicircular canals. ${ }^{11}$ This relationship between the plane of a semicircular canal and the eye movements resulting from its activation is supported by the observation that the nystagmus due to benign paroxysmal positional vertigo affecting the posterior canal is in the plane of that canal. ${ }^{12}$

The direction and alignment of nystagmus noted after hyperventilation can be used to evaluate the hypothesis that these eye movements result from a specific effect, either excitation or inhibition, on vestibular-nerve afferents arising from individual semicircular canals. The vestibular nerve has superior and inferior divisions. Vestibular schwannomas can arise from either the superior division of the vestibular nerve (through which pass fibers that innervate the ampullae of the horizontal and superior semicircular canals as well as the utriculus) or inferior division (through which pass fibers that innervate the ampullae of the posterior semicircular canal and the sacculus). The planar characteristics of the nystagmus elicited by hyperventilation would be expected to correlate with the location of the tumor.

We used three-dimensional eye movement recording techniques to study the nystagmus induced by hyperventilation in six patients with unilateral vestibular schwannoma. Vestibular symptoms were the dominant complaints at the time of presentation in five of these patients. The evoked nystagmus in each case was a "recovery" nystagmus-its direction could

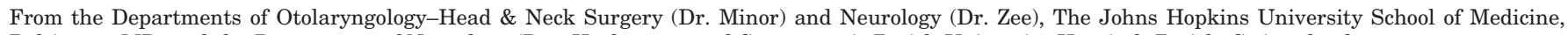
Baltimore, MD; and the Department of Neurology (Drs. Haslwanter and Straumann), Zürich University Hospital, Zürich, Switzerland.

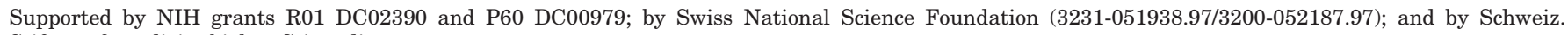
Stiftung f. medizin.-biolog. Stipendien.

Received February 11, 1999. Accepted in final form July 28, 1999.

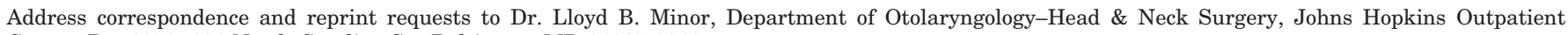
Center, Rm 6253, 601 North Caroline St., Baltimore, MD 21287-0910. 

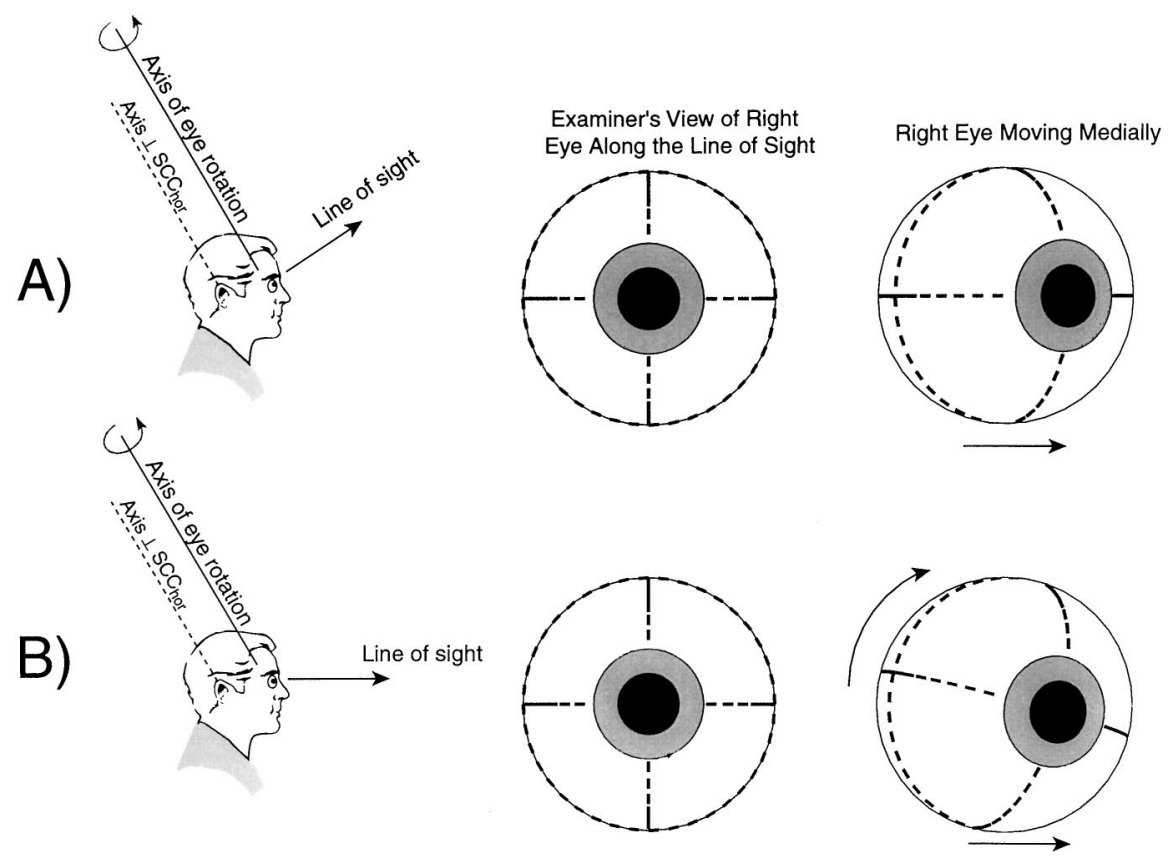

be predicted based upon excitation of specific ampullary nerves innervating the semicircular canals of the affected labyrinth. The relationship between the planes of the semicircular canals and the eye movements recorded in each patient enabled us to determine the effects of hyperventilation on fibers innervating each canal. The location of the tumor noted at the time of surgical resection corresponded to the ampullary nerves that were predicted to be affected based upon the eye movements evoked by hyperventilation.

Methods. Clinical evaluation. Six patients with nystagmus induced by hyperventilation in whom a vestibular schwannoma was subsequently found on gadoliniumenhanced cranial MRI were studied. These patients were identified over an 18-month period from the Vestibular Disorders Clinics of the Departments of Otolaryngology and Neurology at the Johns Hopkins Medical Institutions. A complete neuro-otological examination was performed in each patient. This evaluation included assessment of spontaneous and head-shaking-induced nystagmus, vestibulo-ocular responses to rapid head movements, and observation of eye movement responses to hyperventilation. For the responses to rapid head movements, patients were instructed to look at the examiner's face while rapid, high-acceleration impulses were delivered to the left and right. ${ }^{13} \mathrm{~A}$ normal response consisted of a vestibuloocular reflex that began at an almost imperceptible latency after the onset of the head movement and kept gaze stable during the head movement. For the other tests, patients sat in a dimly illuminated room and wore Frenzel goggles. They hyperventilated for 30 to 40 seconds, taking an average of one deep breath per second.

The directional conventions used for reporting torsional nystagmus merit clarification. It is customary to describe the direction of horizontal and vertical eye movements from the patient's point of regard. We have followed this same convention in the description of torsional eye move-
Figure 1. The eye movements elicited by stimulation of a horizontal semicircular canal will, when viewed by an examiner, appear to have differing horizontal and torsional components according to the position of the eye in the orbit. In each case, the axis of eye rotation is assumed to lie parallel to an axis that is perpendicular to the horizontal semicircular canal. (A) The subject is looking upward such that the line of sight is approximately perpendicular to the axis of eye rotation. In this position, the medial motion of the right eye produced by excitation of the right horizontal canal will appear to the examiner as a horizontal eye movement. (B) The subject is looking straight ahead and the line of sight lies at an oblique angle relative to the axis of eye rotation. In this position, medial motion of the right eye will appear as a horizontal-torsional eye movement. ments. Thus, a clockwise torsional eye movement is defined as movement of the upper pole of the eyes to the patient's right for observations made on clinical examination and for the search coil recordings.

Orientation of the subjects' head and eyes also has an influence on the apparent directional characteristics of the nystagmus that is noted on clinical examination of eye movements with Frenzel goggles. An example of such a dependence of the apparent direction of nystagmus on the position of the eyes in the orbit is provided by posterior canal benign paroxysmal positional vertigo (BPPV). In the eye-fixed coordinate system that is implicitly used to describe eye movements on clinical examination, the nystagmus of posterior canal BPPV appears to be vertical (upbeating) and torsional (superior poles of the eyes beating toward the affected ear) when the eyes are centered in the orbit. ${ }^{5}$ The relative vertical and torsional components of the nystagmus are accentuated when the subjects' eyes are deviated away from and toward the affected ear, respectively. These same eye movements would show no dependence on eye position if represented in a coordinate system that was fixed with respect to the head or to the surrounding space such as an earth-fixed field coil system.

The relationship between position of the eyes in the orbit and the apparent directional characteristics of eye movements observed on clinical examination for the nystagmus expected from horizontal canal stimulation are shown in figure 1. The subject's eyes are deviated upward in figure $1 \mathrm{~A}$ such that the line of sight is approximately parallel to the plane of the horizontal canals. In this case, the axis of rotation of the eye corresponds approximately to an axis perpendicular to the horizontal semicircular canals. As the eye moves medially, the motion as seen by the examiner is horizontal as shown in the diagram. The subject is looking straight ahead (eyes centered in the orbit) in figure $1 \mathrm{~B}$, and the line of sight forms an oblique angle with respect to an axis of rotation that is perpendicular to the horizontal semicircular canals. As the eye moves medially, the resulting eye movement that is observed by the exam- 
Table 1 Maximum slow phase eye velocity of hyperventilation-induced nystagmus

\begin{tabular}{lcrrrr}
\hline Patient & Side of tumor & $\mathrm{EV}_{\text {tot }}$ & $\mathrm{EV}_{\text {hor }}$ & $\mathrm{EV}_{\text {ver }}$ & $\mathrm{EV}_{\text {tor }}$ \\
\hline 1 & Left & 27.3 & -18.6 & -2.7 & 20.2 \\
2 & Right & 27.6 & 22.8 & -3.4 & -15.7 \\
3 & Left & 11.7 & -3.3 & -3.8 & 11.4 \\
4 & Left & 33.9 & -22.6 & -17.7 & 21.8 \\
5 & Left & 5.6 & -3.0 & -1.8 & 5.2 \\
6 & Right & 10.5 & -6.5 & 5.2 & -6.9 \\
\hline
\end{tabular}

$\mathrm{EV}_{\text {tot }}$ is the magnitude of the total eye velocity vector and will always be positive. The horizontal, vertical, and torsional components are given as follows: horizontal component $\left(\mathrm{EV}_{\text {hor }}\right)$, positive for leftward, negative for rightward; vertical component $\left(\mathrm{EV}_{\mathrm{ver}}\right)$, positive for downward, negative for upward; torsional component $\left(\mathrm{EV}_{\text {tor }}\right.$ ), positive for clockwise (motion of the upper pole of the eyes to the patient's right), negative for counterclockwise (motion of the upper pole of the eyes to the patient's left).

iner has both horizontal and torsional components. If the subject were able to look downward such that the line of sight was parallel to an axis that was perpendicular to the plane of the horizontal canals, then exactly the same eye movement would appear to be entirely torsional without any horizontal component.

Three-dimensional eye movement recordings. Hyperventilation-induced responses were also recorded with three-dimensional scleral search coil techniques. Patients gave informed consent for scleral search coil recording through a protocol approved by the Joint Committee on Clinical Investigation of The Johns Hopkins University School of Medicine. They were in darkness during and after the hyperventilation and were instructed to keep their eyes open. Horizontal, vertical, and torsional components of the movement of the left eye were recorded using a dual search coil imbedded in a silicone annulus that was placed around the cornea (Skalar; Delft, the Netherlands). The eye was anesthetized with topical proparacaine before placement of the search coil. The field coil consisted of a cubic coil frame of welded aluminum with a side length of $1.02 \mathrm{~m}$. Three orthogonal magnetic fields (frequencies: 55.5, 83.3, $42.6 \mathrm{kHz}$; intensity: 0.088 Gauss) were produced by the system. Synchronous detection was used to identify amplitude-modulated signals. The bandwidth was 0 to 90 $\mathrm{Hz}$. The calibration procedures for the search coil technique have been described previously. ${ }^{14}$

During the recording sessions subjects sat in darkness inside the coil frame so that the center of the interpupillary line coincided with the center of the frame. Orientation of the head was maintained with the use of a bite bar that was oriented horizontally such that Reid's line (the line connecting the lower rim of the orbit with the center of the external auditory canal) was pitched about $20^{\circ}$ nose up with respect to earth horizontal. This head position brought the horizontal semicircular canal approximately $42^{\circ}$ nose up relative to the earth-horizontal plane. Subjects were instructed to look straight ahead during the times that hyperventilation-induced nystagmus was being recorded.

The three-dimensional eye movements were expressed as angular velocity vectors. Data were displayed relative to a three-dimensional Cartesian coordinate system that was defined such that the $\mathrm{x}$-axis pointed forward, the $\mathrm{y}$-axis to the left, and the z-axis upward. Angular velocity vector components along the $\mathrm{x}-, \mathrm{y}-$, and $\mathrm{z}$-axis thus corresponded to torsional, vertical, and horizontal eye movements, respectively. The following direction conventions for eye movements in a positive direction are established by this coordinate system: clockwise (as viewed from the subject's perspective), downward, and leftward. Conversely, counterclockwise, upward, and rightward eye movements are negative.

Saccades were identified on the eye velocity records with an algorithm developed by Holden et al. ${ }^{15}$ These saccades were then removed and linear interpolation based upon eye velocity before and after the saccade was used to obtain a continuous representation of slow-phase eye velocity. Removal of saccades was verified by visual inspection.

Because the field coils were earth-fixed, the position of the head within the field had an influence on the relative horizontal, vertical, and torsional components of the eye movements that are reported in table 1 . This effect was taken into consideration in the analysis of the data. We projected the eye velocity vectors into a coordinate system that was tilted $42^{\circ}$ upward so that the horizontal canals approximately align with the new $x$-y plane of the rotated coordinate system. This, in effect, aligns the horizontal canals with gaze when the subject looks straight ahead. To determine which semicircular canals contribute to the eye movements elicited by hyperventilation, we assumed that the eye velocities were caused exclusively by the activity of the vestibular nerves on the side of the tumor, and that eye velocities due to activation of more than one canal were a linear superposition of eye velocities produced by each individual canal. The estimated orientation of the semicircular canals during the eye movement recording was determined by the stereotaxic orientation of the canals in the head as reported by Blanks et al. ${ }^{16}$ and the orientation of the head (Reid's line) with respect to the magnetic field.

We recorded nystagmus evoked by hyperventilation and used the velocity of the slow-phase components of this nystagmus to calculate the corresponding stimulation of the semicircular canals. We assumed that the same relation existed between the evoked eye movements and canal stimulation as for the vestibulo-ocular reflex. Thus, projection of the inverse of the eye velocity vector onto the canal vectors ipsilateral to the side of the tumor should yield the pattern of stimulation of the canals. In the calculations, we assumed a small shift between the eye velocity vector and the vector describing the orientation of the canals. This shift was necessitated by the nonorthogonal relationship of the semicircular canals. Positive projections corresponded to canal excitation, and negative projections to canal inhibition. The overall contribution of each canal was determined by integrating the respective canal contributions for eye velocities exceeding $2 \%$ second. To calculate the relative contribution of each canal the resulting components were then normalized by dividing each component by the "length" of the overall stimulation

$$
\sqrt{\text { stim }_{\text {hor }}^{2}+\text { stim }_{\text {sup }}^{2}+\text { stim }_{\text {post }}^{2}}
$$

Torsional eye movements elicited by vestibular stimulation have a lower gain than horizontal or vertical eye movements. ${ }^{17}$ We performed our calculations for the rela- 
Table 2 Relative contribution of the individual ampullary nerves on the side of the tumor to the hyperventilation-induced nystagmus

\begin{tabular}{lccc}
\hline Patient & Horizontal & Superior & Posterior \\
\hline 1 & 0.87 & 0.45 & 0.19 \\
2 & 0.94 & 0.34 & 0.01 \\
3 & 0.62 & 0.69 & 0.38 \\
4 & 0.77 & 0.64 & 0.02 \\
\hline
\end{tabular}

The algorithm used for projection of the eye velocity vector into canal vectors is described in Methods.

tive canal contributions based upon a horizontal and vertical gain of 1.0 and torsional gain of $1.0,0.66$, or 0.5 . These calculations yielded similar results with no difference in the dominant canal contribution determined based upon the assumed torsional gain. Results of the canal plane projections based upon a torsional gain of 0.5 are reported in table 2. For small responses, this decomposition was strongly affected by the slight variability in the eye velocity traces. Therefore, we only performed these canal-plane projections when the maximum total eye velocity exceeded $11 \%$ second.

Caloric and rotatory chair testing. Caloric tests were performed with a temperature switch irrigation technique for $30.5^{\circ}$ and $43.5^{\circ} \mathrm{C} .{ }^{18}$ Horizontal eye movements were recorded with electro-oculography, and maximum velocity of the slow-phase components of nystagmus evoked by each ear was analyzed for unilateral weakness and directional preponderance as determined by the conventional
Jongkees formulae. ${ }^{19}$ Responses were reported as normal if the slow-phase components of the nystagmus evoked by each irrigation had a velocity of $>8 \%$ second and if the asymmetry between responses for the left and right ears was $<20 \%$. Asymmetries greater than this value were reported as a percentage for unilateral vestibular weakness.

Rotatory chair testing was performed with velocity steps to 60 and $240 \%$ second in the rightward and leftward directions while subjects were in darkness. The time constant ( $\mathrm{Tv}$ ) was measured from responses to the $60^{\circ} /$ second rotations as the time at which eye velocity had declined to $37 \%$ of its initial value. Gain (Gv) was measured from responses to $240 \%$ second velocity steps as the ratio of maximum horizontal slow-phase eye velocity divided by the stimulus velocity. Gv was typically measured for rotations given in two positions: in the plane of the horizontal canals and elevated $60^{\circ}$ in the head-up position. Tusa et al. ${ }^{20}$ have shown that rotations in the plane of the horizontal canals are more likely to identify asymmetries in Gv after unilateral ablation of vestibular function.

Results. Clinical examination and testing. A nystagmus induced by hyperventilation was identified in six patients with vestibular schwannoma. In each case, the nystagmus was visible on clinical examination with Frenzel goggles. The vestibular symptoms (dizziness and dysequilibrium) that led these patients to be referred for evaluation were either reproduced or exacerbated by hyperventilation. Table 3 gives the symptoms and signs noted at the time of presentation for the six patients in the study. Complaints related to balance prompted the referral of five of these six patients for evaluation at our tertiary

Table 3 Symptoms and signs in patients with hyperventilation-induced nystagmus and vestibular schwannoma

\begin{tabular}{|c|c|c|c|c|c|}
\hline \multirow[b]{2}{*}{ Patient } & \multirow[b]{2}{*}{ Age, $y$} & \multirow[b]{2}{*}{ Sex } & \multirow[b]{2}{*}{ Symptoms } & \multicolumn{2}{|c|}{ Signs } \\
\hline & & & & General & $\begin{array}{l}\text { Hyperventilation- } \\
\text { induced nystagmus } \\
\text { slow phases }\end{array}$ \\
\hline 1 & 40 & M & 1-y history of dizziness and nausea & Normal & Right, up, clockwise \\
\hline 2 & 62 & M & $\begin{array}{l}\text { 10-mo history of dizziness and } \\
\text { imbalance; rotatory vertigo with } \\
\text { exertion as when playing tennis }\end{array}$ & $\begin{array}{l}\text { Spontaneous nystagmus, } \\
\text { rightward; horizontal head } \\
\text { shaking nystagmus, } \\
\text { rightward; vertical head } \\
\text { shaking nystagmus, } \\
\text { leftward }\end{array}$ & $\begin{array}{l}\text { Left, up, counter- } \\
\text { clockwise }\end{array}$ \\
\hline 3 & 62 & M & $\begin{array}{l}\text { Dysequilibrium and progressive } \\
\text { gait ataxia }\end{array}$ & $\begin{array}{l}\text { Left head tilt; diminished } \\
\text { VOR noted with head } \\
\text { thrusts to left; spontaneous } \\
\text { nystagmus: rightward, } \\
\text { clockwise slow phases on } \\
\text { left and down gaze }\end{array}$ & Right, up, clockwise \\
\hline 4 & 64 & M & $\begin{array}{l}\text { Unsteadiness and dysequilibrium } \\
\text { increasing in severity over } 2 \frac{1}{2}-\mathrm{y} \\
\text { and worse on exertion }\end{array}$ & $\begin{array}{l}\text { Horizontal head shaking } \\
\text { nystagmus, leftward }\end{array}$ & Right, up, clockwise \\
\hline 5 & 52 & $\mathrm{~F}$ & $\begin{array}{l}\text { Left tinnitus and sensorineural } \\
\text { hearing loss }\end{array}$ & Normal & Right, up, clockwise \\
\hline 6 & 49 & $\mathrm{~F}$ & Episodic vertigo & Normal & $\begin{array}{l}\text { Right, down, } \\
\text { counterclockwise }\end{array}$ \\
\hline
\end{tabular}

The direction of the nystagmus refers to the slow-phase components.

VOR $=$ vestibulo-ocular reflex 
Table 4 Findings on diagnostic studies in patients with hyperventilation-induced nystagmus and vestibular schwannoma

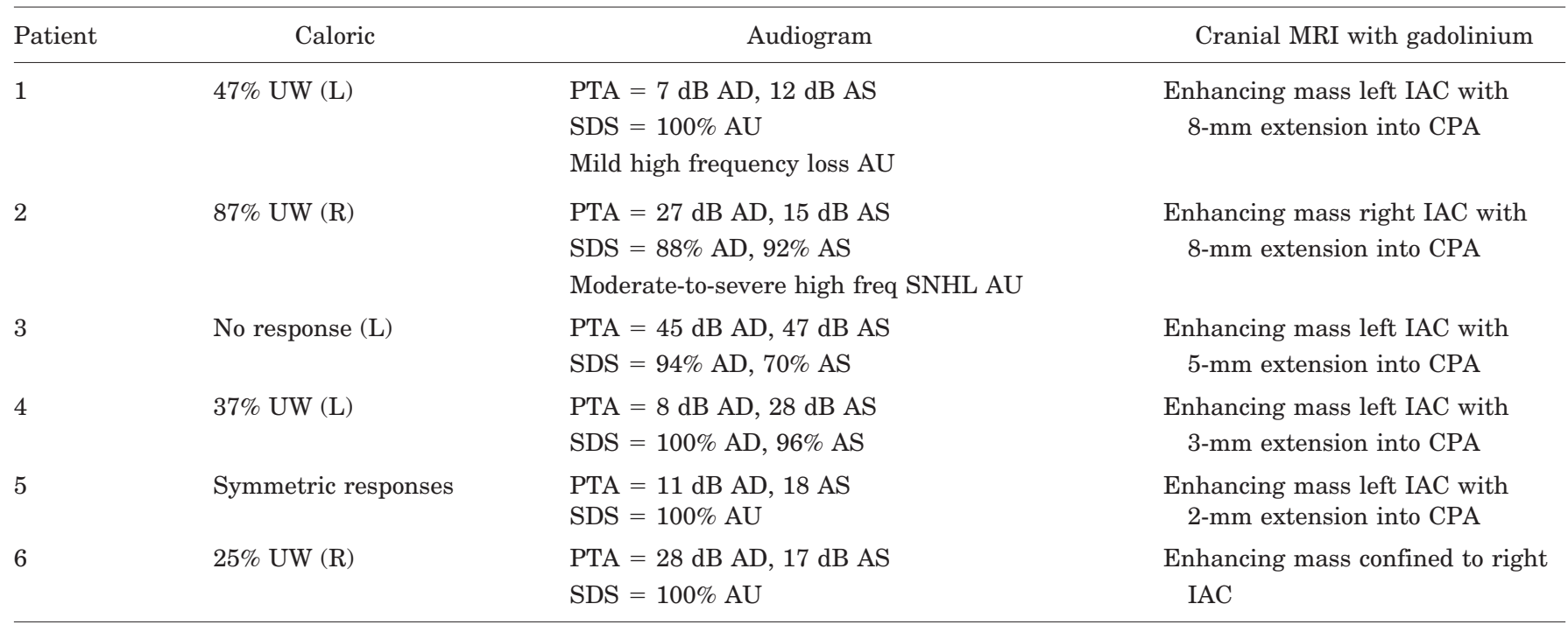

The quantitative interpretation of the results of caloric tests is described in the Methods section (UW, unilateral weakness). Audiometric results are reported as pure tone average (PTA), the average of hearing thresholds at 500, 1,000, and 2,000 Hz; speech discrimination score (SDS), the percentage of words from a phonetically balanced list that are repeated correctly by the listener; sensorineural hearing loss (SNHL); right ear (AD), left ear (AS), and both ears (AU). Normal audiometric values: PTA $\leq 20 \mathrm{~dB}$; SDS $\geq 85 \%$.

$\mathrm{IAC}=$ internal auditory canal; CPA $=$ cerebellopontine angle.

care center. Only one subject (Patient 5) had an auditory complaint (left tinnitus) that would have specifically raised suspicion of a retrocochlear process such as vestibular schwannoma.

Three patients had signs on clinical examination of unilateral vestibular hypofunction. These signs were a spontaneous nystagmus and head-shaking-induced nystagmus indicative of right vestibular hypofunction in Patient 2, a head-thrust sign and spontaneous nystagmus indicative of left vestibular hypofunction in Patient 3, and a headshaking-induced nystagmus indicative of left vestibular hypofunction in Patient 4.

The hyperventilation-induced nystagmus observed on examination with Frenzel goggles typically had horizontal, vertical, and torsional components (see table 3 ). The horizontal and torsional components were more prominent than the vertical component. As described in the Methods section, the direction of the observed eye movement depends upon the position of the eye in the orbit. Figure 1B depicts the typical orientation of the patient's line of sight during the times that these examinations were made.

The findings on caloric tests, audiometry, and cranial MRI are summarized in table 4. All patients except Patient 5 had diminished caloric responses on the side of the tumor. Pure tone thresholds and speech audiometry were symmetric in Patient 5 and revealed only slight asymmetries between the two ears in Patients 1, 2, and 6. Asymmetries measuring greater than $20 \mathrm{~dB}$ in pure tone thresholds or greater than $20 \%$ in speech discrimination scores were noted in Patients 3 and 4. Each tumor was noted to fill the internal auditory canal on gadoliniumenchanced MRI. The size of the tumor was reported as the widest dimension measured from the portion of the tumor extending into the cerebellopontine angle. ${ }^{21}$

The data from rotatory chair tests are presented in table 5 . For the four patients in whom rotatory chair tests were obtained before surgical resection of the vestibular schwannoma, an asymmetry measuring $>15 \%$ was noted for time constant in two (Patients 1 and 5) and for gain in one (Patient 2). The time constant was diminished for rotations toward the side of the tumor in Patient 1 . The small gain asymmetry that was noted in this patient resulted from responses being lower in amplitude for rotations toward the side of the tumor, but only in the head-up position. The gains measured in the head-down position in this patient were more symmetric. Patient 2 had an asymmetry in time constant and in gain, measured in the headdown position, with rotations toward the side of the tumor leading to lower responses. The time constants for rotations were $<10$ seconds for rotations in both directions in Patients 2 and 3, a finding associated with vestibular dysfunction but not localizing with respect to side or etiology. ${ }^{20}$ Patient 5 had an asymmetry in time constants (shorter for rotations toward the side of the tumor) but not in gains.

Two subjects (Patients 2 and 4) had undergone cranial MRI without gadolinium enhancement before referral to our center for evaluation. In each case, these nonenhanced MRIs were interpreted as normal. A gadolinium-enhanced cranial MRI in these patients and the others in the study revealed a mass in the internal auditory canal with differing extension into the cerebellopontine angle as described in table 4. An axial section from such an MRI in Patient 4 is shown in figure 2 .

Search coil recording of eye movements. The nystagmus in response to hyperventilation that was recorded with the three-dimensional scleral search coil technique in Patient 1 is shown in figure 3 . The eye velocity vectors have been projected into a coordinate system that was tilted $42^{\circ}$ upward as described in the Methods section. The response after this coordinate transformation consists of mainly a horizontal nystagmus with small vertical and 


\begin{tabular}{|c|c|c|c|c|c|c|c|c|c|c|}
\hline \multirow[b]{2}{*}{ Patient } & \multirow[b]{2}{*}{ Test } & \multicolumn{3}{|c|}{$60 \% \mathrm{~s}$} & \multicolumn{3}{|c|}{$240^{\circ} / \mathrm{s} \mathrm{HU}$} & \multicolumn{3}{|c|}{$240 \% \mathrm{~s} \mathrm{HD}$} \\
\hline & & $\mathrm{T}_{\mathrm{R}}$ & $\mathrm{T}_{\mathrm{L}}$ & DP (\%) & $\mathrm{G}_{\mathrm{R}}$ & $\mathrm{G}_{\mathrm{L}}$ & DP (\%) & $\mathrm{G}_{\mathrm{R}}$ & $\mathrm{G}_{\mathrm{L}}$ & $\mathrm{DP}(\%)$ \\
\hline 1 & Post & 10.1 & 15.7 & $21.7(\mathrm{~L})$ & 0.62 & 0.78 & $11.3(\mathrm{~L})$ & 0.65 & 0.77 & $7.8(\mathrm{~L})$ \\
\hline 2 & Pre & 7.7 & 5.1 & $18.5(\mathrm{R})$ & 0.27 & 0.24 & $5.8(\mathrm{R})$ & 0.35 & 0.24 & $19.3(\mathrm{R})$ \\
\hline 5 & Pre & 11.6 & 6.4 & $29.1(\mathrm{R})$ & 0.62 & 0.63 & $0.7(\mathrm{~L})$ & 0.64 & 0.71 & $5.3(\mathrm{~L})$ \\
\hline
\end{tabular}

The measurement techniques used for determining gain and time constant are described in Methods. Data are given from tests obtained before surgical resection of the tumor (Pre) and at 6 weeks (Patient 6) and 5 weeks (Patient 4) after tumor resection (Post). Time constants and gains were calculated from responses to velocity-step rotations that reached a peak velocity of 60 and $240 \%$, respectively. The responses to $240 \%$ s rotations were measured with the head tilted downward such that the horizontal canals were approximately in the plane of rotation (HD) and elevated such that the horizontal canals were tilted $60^{\circ}$ upward relative to the plane of rotation (HU). The direction of the time constant and gain measurements are reported relative to the motion of the eye. Directional preponderance (DP) is reported as the percent difference between responses to rightward and leftward rotations. $T_{R}$ and $T_{L} ; G_{R}$ and $\mathrm{G}_{\mathrm{L}}$, time constants and gains for eye rotations to the right and left, respectively. Note that rightward rotation of the eye is caused by leftward rotation of the head and vice versa. Normal findings on rotatory chair: Time constant, 10-30 s; Gain $>0.4$.

torsional components. Note that the same data would exhibit a large torsional velocity component if expressed in the original coordinate system.

The maximum total eye velocity of this nystagmus as well as the maximum velocity of the horizontal, vertical, and torsional components determined from the threedimensional eye movement recordings are given in table 1. The vector representing the maximum eye velocity in response to hyperventilation was projected into the planes of the horizontal, anterior, and posterior canals in table 2 . Patients 5 and 6 were not included in this analysis because their maximum responses were lower than that required to identify the projection clearly.

The nystagmus began 5 to 15 seconds after the onset of hyperventilation and the velocity of the slow-phase components reached a maximum 40 to 60 seconds later. The horizontal, vertical, and torsional components of the nystagmus began simultaneously in five of the patients. In all patients, the nystagmus declined over the course of 40 to 60 seconds after the maximum velocity had been reached. A reversal in the direction of the nystagmus was then noted in Patients 2, 3, and 5. Figure 4 shows the nystagmus reversals (indicated by arrows on the horizontal trace) for Patient 2. This patient was the only one to have had more than one reversal component in the response. The eye velocity in figure 4 is displayed before projection into a coordinate system aligned with the semicircular canals.

The magnitude and direction of eye velocity showed little dependence on eye position. A linear regression of maximum eye velocity with respect to eye position for the six patients in the study revealed the following correlation coefficients for horizontal, vertical, and torsional eye position, respectively: $0.66,-0.72$, and 0.23 . The findings suggest that there may be a weak relationship between horizontal and vertical eye position and maximum velocity of the response $(p>0.2$, analysis of variance for regressions). The variation in mean eye position around the time of maximal eye velocity tended to be small. Inspection of the eye position and velocity records provided further evidence that, when represented in a head- or space-fixed coordinate system, the response to hyperventilation showed little variation with eye position. In Patient 1 , there was a change in horizontal eye position of about $11^{\circ}$ over a 3-second period of the record, but the horizontal eye velocity was only slightly decreased from 16 to $14 \%$ second and the vertical velocity remained constant at $17 \%$ second. Similarly, in Patient 4 the vertical eye position changed by about $12^{\circ}$ over a 5 -second period of the record, but the velocity components were unaffected.

Surgical resection of the tumor. Each of the patients underwent surgical removal of the vestibular schwannoma through a suboccipital approach. The tumor was noted to arise from the superior vestibular nerve in each case but also appeared to involve the inferior vestibular nerve in Patients 3 and 4. The resection of the tumor was judged to be complete in each case. In Patient 1 , the tumor was dissected off the superior vestibular nerve with minimal disruption of the nerve fibers that were not directly involved. Caloric tests performed 6 weeks after surgery showed a $34 \%$ reduction of caloric responses in the left ear compared to the right. The rotatory chair test performed at this same time (table 5) showed a reduction in time constants in comparison to preoperative findings. The time constant was shorter for rotations toward the side from which the tumor had been removed. The gains measured in this patient remained normal in magnitude and symmetric.

The hyperventilation-induced nystagmus was eliminated by surgical resection of the tumor in four of the six patients. Complete removal of the tumor in Patient 4 required surgical sectioning of both the superior and inferior vestibular nerves. There was no response to an ice water caloric stimulus and rotatory chair testing showed reduced gains for rotations toward the lesioned side on studies performed five weeks postoperatively. A severe-toprofound hearing loss was present in the operated ear after surgery. The hyperventilation-induced nystagmus that was noted in this patient after resection of the tumor was directed as before the procedure, but its amplitude was substantially reduced. Patient 6 was noted to have a hyperventilation-induced nystagmus with the following components when tested 10 days after resection of the 


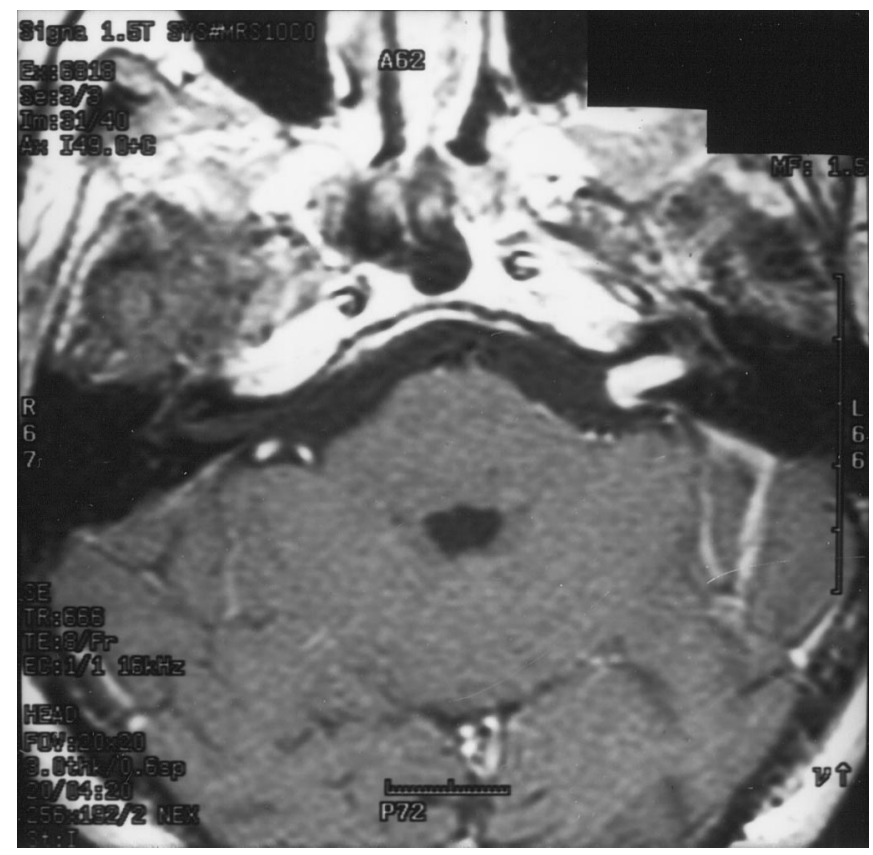

Figure 2. Axial image at the level of the internal auditory canals of a gadolinium-enhanced, T1-weighted MRI in Patient 4. Note the enhancing mass within the left internal auditory canal.

tumor: horizontal $=-11.7$, vertical $=-6.9$, and torsional $=11.4 \%$ second. When projected into canal planes, this nystagmus corresponds to inhibition of the right horizontal (0.86) and posterior (0.50) canals.

Discussion. Clinical features. We have identified six patients with vestibular schwannoma in whom nystagmus was induced by hyperventilation. The symptoms leading to a neuro-otology consultation in Patients 2 and 4 reflected abnormalities in vestibular function resulting from exercise and hyperventilation. Patients 1 through 4 and 6 had vestibular symptoms preceding or of greater severity than auditory symptoms. An asymmetric or unilateral sensorineural hearing loss, often with a preferential effect on the higher frequencies, is the most common initial symptom and finding in patients with vestibular schwannoma. ${ }^{22,23}$ Lustig et al. ${ }^{24}$ found normal or symmetric audiometry in 29 of $546(5 \%)$ patients with vestibular schwannoma. Dizziness, vertigo, or dysequilibrium were the most common presenting complaints in those patients without a localizing abnormality on audiometry. Testing for hyperventilationinduced nystagmus was not performed.

Findings from vestibular tests assisted in localizing and defining the vestibulopathy. Caloric tests revealed vestibular hypofunction in the ear with the vestibular schwannoma in five of the six cases. The caloric response was absent in the affected ear in only one case (Patient 3). Asymmetries in gain or time constant on rotatory chair testing that localized the side of the abnormality could be discerned in three of the four patients that were tested before resection of the tumor. In each case, the asymme- tries were larger for caloric than for rotatory chair tests.

Two patients (Patients 2 and 4) had undergone cranial MRI without gadolinium enhancement before referral to our center for evaluation. In each case, these nonenhanced MRIs were interpreted as normal. A gadolinium-enhanced cranial MRI in each of these patients revealed a mass in the internal auditory canal. These findings indicate that the presence of hyperventilation-induced nystagmus should prompt evaluation with either a gadoliniumenhanced MRI of the brain and internal auditory canals or an MRI technique with equivalent sensitivity for detecting a mass in the internal auditory canal. ${ }^{25-27}$ Until studies examining the incidence of nystagmus induced by hyperventilation are performed in large numbers of patients with known vestibular pathology, including vestibular schwannoma, the absence of hyperventilation-induced nystagmus should not influence a decision about obtaining an imaging study based upon other clinical findings or suspicions.

Removal of the vestibular schwannoma eliminated the hyperventilation-induced nystagmus in four of the six cases. In Patient 4, the schwannoma was completely resected and both the superior and inferior vestibular nerves were sectioned. There was no response to a caloric stimulus on the operated side after the operation. A hyperventilation-induced nystagmus of lower amplitude but with the same directional characteristics was noted postoperatively. This nystagmus may have been caused by excitation of vestibular fibers coursing with the cochlear nerve, which was anatomically preserved during the procedure. Previous anatomic studies have shown that up to $25 \%$ of vestibular fibers may course within the structure that on gross inspection appears to be entirely the cochlear nerve. ${ }^{28}$ It is not known from which vestibular end organ(s) these fibers arise.

In Patient 6, the nystagmus before resection of the tumor was low in amplitude but reflected inhibition of the right horizontal canal and excitation of the right posterior canal. This unusual pattern of mixed excitation and inhibition of individual canals was not seen in any of the other patients. After resection of the tumor, the hyperventilation-induced nystagmus reflected strong inhibition of the right horizontal and posterior canals. Such an effect could be attributable to accentuation of the underlying spontaneous nystagmus..$^{1,3}$

Hyperventilation has been reported to accentuate an underlying spontaneous nystagmus in patients following vestibular neurectomy. ${ }^{1,3}$ An "ablative" nystagmus, horizontal and torsional slow-phase components directed toward the neurectomized side, was noted in these patients. The nystagmus induced by hyperventilation in the current study was a "recovery" nystagmus in Patients 1 through 5 -i.e., it reflected excitation on the side of the tumor. Bance et al. ${ }^{3}$ found hyperventilation-induced nystagmus in 23/28 (82\%) patients with vestibular schwannoma. 

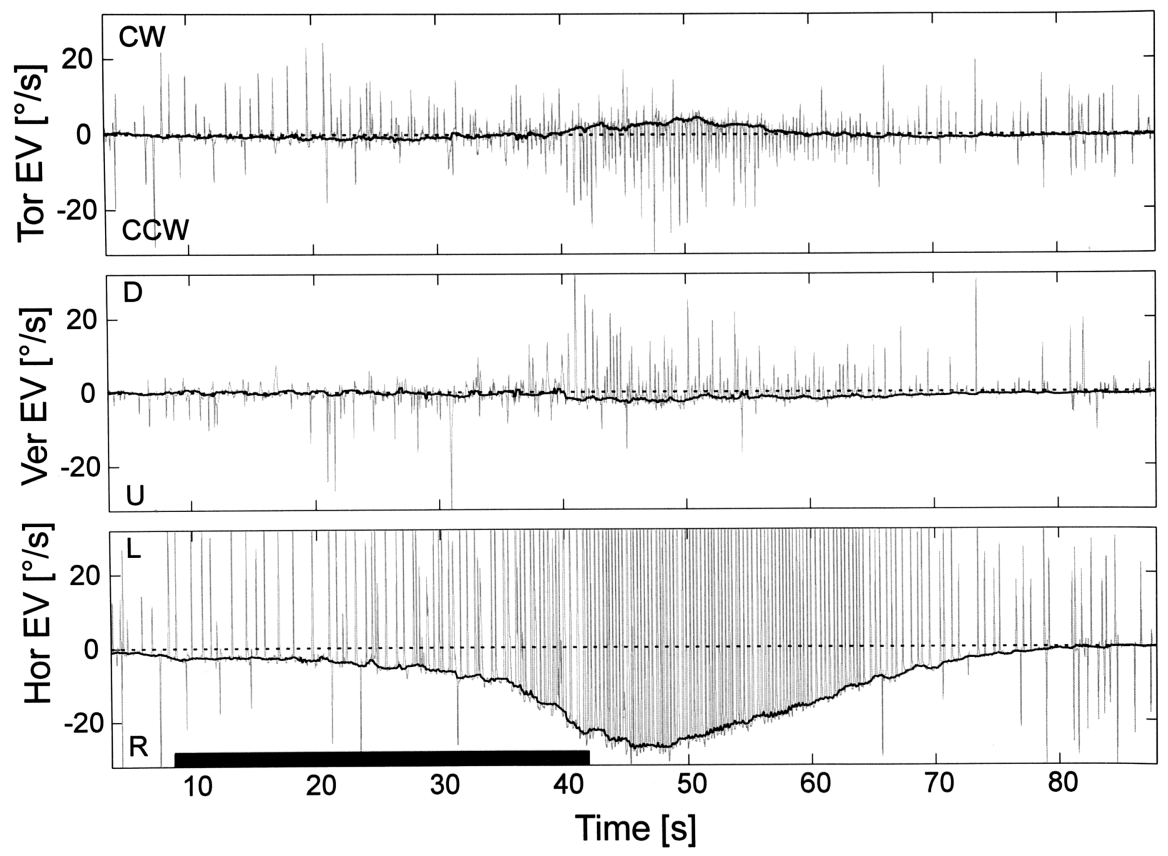

Figure 3. Nystagmus evoked by hyperventilation in Patient 1, who had a vestibular schwannoma on the left side. The period of hyperventilation is indicated by the dark bar on the lower abscissa. Torsional, vertical, and horizontal angular velocity vector components of the response are displayed. The desaccaded eye velocity signal is shown by the darker trace on each record, and the fast phases are indicated by the lighter gray. Positive values for the torsional, vertical, and horizontal components correspond to clockwise $(\mathrm{CW})$, downward, and leftward eye movements, respectively. For display and analysis of these data, the eye velocity vectors were projected into a coordinate system that was tilted $42^{\circ}$ upward as described in Methods.
The nystagmus had slow-phase components directed opposite to the side of the tumor similar to the nystagmus noted in the current study (excitatory) in $11 / 23(48 \%)$ and had ipsilaterally directed slowphase components (inhibitory) in $12 / 23$ (52\%). These authors also noted a tendency for smaller tumors (mean size $=1 \mathrm{~cm}$ ) to have an excitatory nystagmus in response to hyperventilation and for larger tumors (mean size $=1.55 \mathrm{~cm}$ ) to have an inhibitory response. Also, an inhibitory nystagmus was more commonly associated with a complete loss of caloric responses on the affected side. The patients in our study had smaller tumors and most had preserved caloric responses; therefore, they corresponded to the group in which Bance et al. more commonly identified a "recovery" nystagmus.

In contrast to our study in which the maximum velocity of the slow-phase components of the nystagmus was $>10 \%$ second in five of the six patients and $>20 \%$ second in three, Bance et al. ${ }^{3}$ reported considerably lower amplitude responses in most of their patients. Only 4 of the 47 patients in which the nystagmus was evaluated quantitatively in the study of Bance et al. had a slow-phase eye velocity $>10 \%$ second. These investigators used infrared videonystagmography to record eye movements whereas search coil techniques were used in our study. The search coil recordings provide a precisely calibrated record of the response with unambiguous representation in three dimensions.

The postoperative findings in Patient 1 provide evidence that ablation of vestibular function is not required for elimination of hyperventilation-induced nystagmus. The tumor in this patient was small and was dissected off of the superior vestibular nerve from which it arose with minimal injury to either division of the vestibular nerve. Postoperative caloric and rotatory chair testing showed that vestibular function remained in the ear from which the tumor was removed. To explain the elimination of hyperventilation-induced nystagmus with preservation of vestibular function in this patient, we suggest that the tumor resulted in demyelination of only a group of vestibular fibers immediately surrounding it. Other fibers within the vestibular nerve were unaffected.

Mechanism of hyperventilation-induced nystagmus. We propose that partial demyelination of the vestibular nerve caused by the schwannoma is the underlying pathology in these cases of hyperventilation-induced nystagmus. The presumed mechanism involves the effects of hyperventilation on the physiology of partially demyelinated axons. Carbon dioxide levels measured transcutaneously have been shown to decrease by an average of $21 \%$ after a 30 -second period of hyperventilation. ${ }^{1}$ The reduction in arterial $\mathrm{PCO}_{2}$ resulting from such a period of hyperventilation has been measured as $6 \mathrm{~mm} \mathrm{Hg}$, which corresponds to $0.8 \mathrm{kPa} .{ }^{29} \mathrm{CSF} \mathrm{pH}$ rises in association with this reduction in $\mathrm{PCO}_{2}$, which leads to a reduction in extracellular ionized $\mathrm{Ca}^{2+}$. Axonal conduction in partially demyelinated nerve fibers improves transiently as a result of the lowering of extracellular $\mathrm{Ca}^{2+} .{ }^{30}$ Support for this mechanism is provided by the reported effects of hyperventilation on physiologic deficits observed in some patients with MS. Hyperventilation or IV administration of $\mathrm{NaHCO}_{3}$ or $\mathrm{Na}_{2} \mathrm{EDTA}$, both of which increase $\mathrm{pH}$ and lower extracellular $\mathrm{Ca}^{2+}$, have been shown to decrease the size of visual scotomas and reduce the abnormal latencies of visually evoked potentials..$^{29-31} \mathrm{We}$ presume that the disappearance of hyperventilation-induced nystagmus after surgical resection of the tumor is due to removal of damaged axons or axonal decompression.

Three-dimensional aspects of the nystagmus. At the bedside, a complete description of nystagmus, 

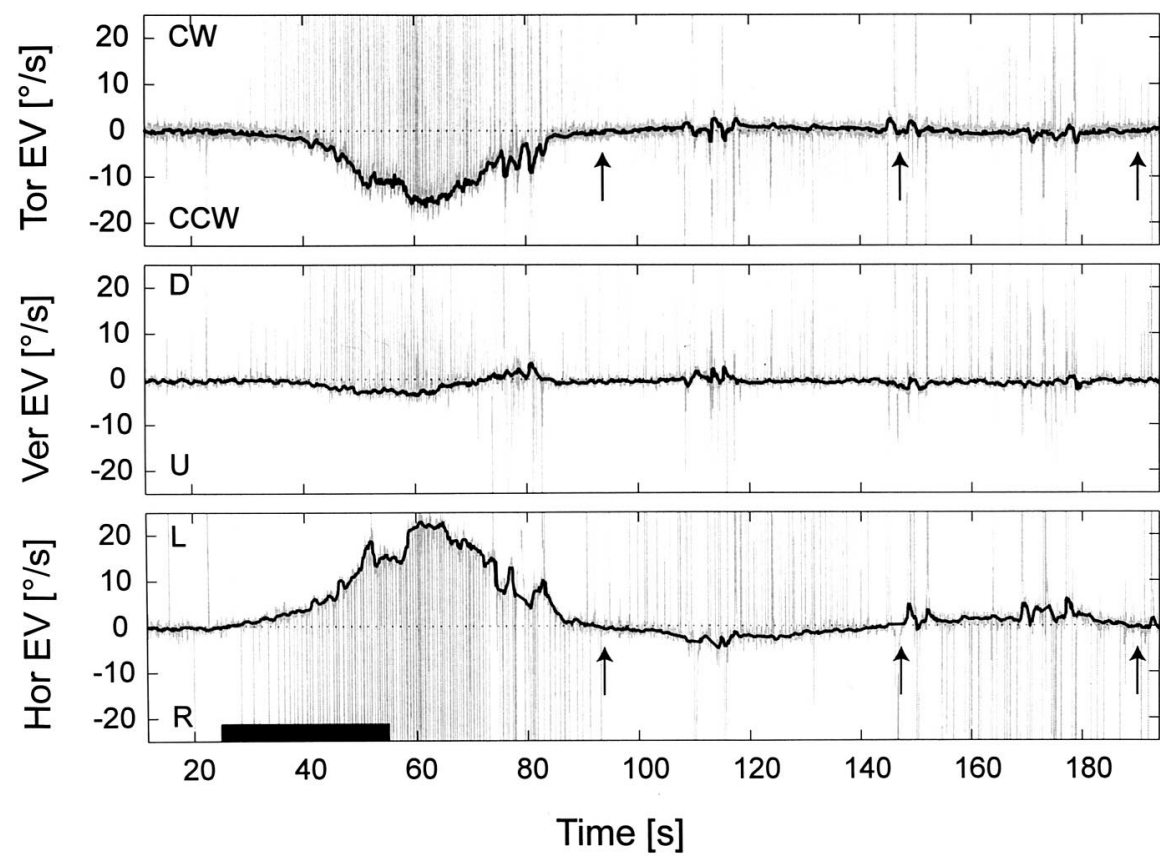

Figure 4. Nystagmus evoked by hyperventilation in Patient 2 with a vestibular schwannoma on the right side. The coordinate reference frame for display of these data are before projection into a coordinate system aligned with the semicircular canals. Note that reversal phases of the response are noted in both the horizontal and torsional components and that the timing of these reversals is coincident for both components. The points of reversal are indicated by the arrows on the figure and can also be identified by the change in direction of the fast-phase components of the horizontal and torsional response. separating it into horizontal, vertical, and torsional components, depends upon the coordinate reference frame used by the observer. This, in turn, is influenced by the vantage point of the observer relative to the direction in which the patient is looking, i.e., the patient's line of sight, and also by the position of the eye of the patient in the orbit. These issues are described in figure 1.

Consider a patient, with the head still, who has a spontaneous jerk nystagmus that is resulting from excitation of the right horizontal canal. The axis of rotation of the eye will be parallel to an axis that is perpendicular to the plane of the horizontal canal. This axis of eye rotation will not change; however, the apparent horizontal and torsional components of the nystagmus seen by the observer will vary with regard to the position of the patient's head, the location of the patient's eye in the orbit, and the vantage point of the observer relative to the patient's line of sight. The observer will note a nystagmus that appears horizontal when the patient's line of sight is perpendicular to the axis of eye rotation (figure 1A). When the patient's line of sight is oriented in more of a straight-ahead position such that there is an oblique angle between the axis of eye rotation and the line of sight, the same nystagmus will appear to have horizontal and torsional components (figure 1B).

During clinical examinations, the observer is describing the nystagmus in an eye-fixed coordinate system. The nystagmus in the example described above is still rotating the eye around the same headfixed axis, even though the horizontal and torsional components that are noted by the observer appear to change with the position of the eye in the orbit. This apparent change in direction is purely related to the coordinate system in which the observations are being made. Once cognizant of these frame-of-reference issues, the observer can perform the necessary men- tal transformations to determine the axis of rotation of nystagmus no matter what the direction of gaze. In the case of the examinations performed with Frenzel goggles in our patients with hyperventilationinduced nystagmus, most had a mixed horizontaltorsional nystagmus when looking straight ahead, which corresponds to rotation around an axis tilted back about $40^{\circ}$ in the head (figure 1B).

The three-dimensional eye movement recordings were initially made with regard to a space-fixed coordinate system which, based upon the orientation of the patient's head in the field coil frame, resulted in the horizontal canals being pitched about $40^{\circ}$ upward relative to the $x-y$ plane of the coordinate system. Display of the nystagmus relative to this orientation would reveal a large torsional component in the eye movements. Expressing the same data with respect to a different coordinate system, which is oriented such that the new $x-y$ plane coincides approximately with the orientation of the horizontal canals, eliminates almost all of the torsion (figure 3). The direction of the remaining torsional component is variable, possibly because the exact orientation of the semicircular canals deviates significantly from the average values used in the evaluation of our data. ${ }^{16}$

The effect of orientation of the head was taken into consideration in the calculation of the relative contributions of each canal to the recorded eye velocities. Patients with a hyperventilation-induced nystagmus of $>11 \%$ second $(n=4)$ showed a consistent pattern (table 4): the contribution of the horizontal and superior canal was larger than that of the posterior canal (one-way ANOVA, $p=0.001$ ). The contribution of the horizontal canal was not larger than that from the superior canal (paired $t$-test, $p=0.17$ ). This result was less pronounced in patients with a small total eye velocity. In Patient 3, where total eye 
velocity was only slightly above the threshold of $11 \%$ second that we have used in our analysis, the contribution of the posterior canal was found to be approximately half that of the horizontal and superior canals. This pattern of excitation is consistent with the fact that all the tumors were on the superior vestibular nerve, which includes the fibers of the horizontal and the superior canals.

The axis of rotation (in a head-coordinate frame) for the hyperventilation-induced nystagmus was relatively independent of the vertical position of the eyes in the head. This finding is somewhat unexpected as a previous study of the vestibulo-ocular reflex evoked by sinusoidal head rotations revealed a dependence of the eye rotation axis on the position of the eye in the orbit..$^{32}$ The eye rotation axes during yaw and pitch rotations tilted in the same direction as the line of gaze but only one-quarter as far. A more complicated relationship between eye position and the axis of eye rotation was found for responses to rapid, high-acceleration impulses of head rotation. ${ }^{33}$ The onset and magnitude of the shift in rotation axis were found to depend upon whether the head movement was active or passive and whether the eye position was upward or downward. It is therefore apparent that the relationship between eye position and the axis of eye rotation may be dependent upon multiple factors associated with the stimulus.

The reversal phases of the responses following hyperventilation indicate that torsion observed during the responses may be principally due to activation of the horizontal canal. There was a close correspondence between the horizontal and torsional components during the secondary and tertiary reversals of the hyperventilation-induced response (figure 4). Such correspondence would not necessarily be predicted if the torsional component of the response were due to vertical canal activation. These reversal phases are likely due to adaptation mechanisms in afferents ${ }^{34}$ and in central processes, ${ }^{35}$ as well as to the recovery processes in the discharge of afferents after hyperventilation.

In our quantitative analysis of the data, we assumed that rotation of the eyes elicited by stimulation of an individual canal is about an axis that is slightly shifted, and not precisely orthogonal to the plane of an individual canal. Such a shift in the axis of rotation is thought to occur in order to compensate for the nonorthogonality of the lateral, superior, and posterior canals. In normal vestibuloocular responses elicited by rotations, the eyes need to move in exactly the same plane as the head for vestibuloocular reflexes to maintain image stability on the fovea during head movements. If the plane of eye movement was defined exclusively by the plane of each stimulated canal, then the nonorthogonal relationship between the canals would result in eye movements that did not align with head movements. These relationships between activation of individual canals and the plane of eye movements are likely to be established in the central pathways that deter- mine activation of extraocular motoneurons based upon signals from the canals. ${ }^{36,37}$ The predicted shift in the axis of eye velocity, based upon stimulation of individual canals, is relatively small for the posterior canal (about $9^{\circ}$ ), but appreciable for the lateral and superior canals (about $23^{\circ}$ ). In the case of the posterior canal, this is reflected in the relatively close alignment between the posterior canal and the plane of rotation of the eye. ${ }^{12,38}$

In our patients, the determination of the contributions of individual canals based upon the alternate assumption-stimulation of a single canal leads to eye movements exactly in the plane of that canaldid not change the dominance of the contribution of the horizontal canal. The contribution from the vertical canals was, however, more variable when such an assumption was made. Because the underlying pathology in all our patients was similar (a schwannoma that predominantly affected the superior division of the vestibular nerve), the profile of canal activation produced by hyperventilation would also be expected to be similar among patients. This prediction was confirmed when the canal activation was determined based upon projections of the eye velocity vector into planes that compensate for the nonorthogonality of canals on the affected side. A direct test of this hypothesis could come from a threedimensional analysis of the nystagmus induced by lateral canal BPPV, as the shift in the eye velocity axis is more prominent for the lateral than for the posterior canal.

\section{References}

1. Sakellari V, Bronstein AM, Corna S, Hammon CA, Jones S, Wolsley CJ. The effects of hyperventilation on postural control mechanisms. Brain 1997;120:1659-1673.

2. Wilson WR, Kim JW. Study of ventilation testing with electronystagmography. Ann Otol Rhinol Laryngol 1981;90:56-59.

3. Bance ML, O'Driscoll M, Patel N, Ramsden RT. Vestibular disease unmasked by hyperventilation. Laryngoscope 1998; 108:610-614.

4. Brackmann DE, Kwartler JA. A review of acoustic tumors: 1983-1988. Am J Otol 1990;11:216-232.

5. Leigh RJ, Zee DS. Diagnosis of central disorders of ocular motility. In: Leigh RJ, Zee DS, eds. The neurology of eye movements, 3rd ed. New York: Oxford University Press, 1999: 405-610.

6. Zee DS, Fletcher WA. Bedside examination. In: Baloh RW, Halmagyi GM, eds. Disorders of the vestibular system. New York: Oxford University Press, 1996:178-190.

7. Walker MF, Zee DS. The effect of hyperventilation on downbeat nystagmus in cerebellar disorders. Neurology 1999 (in press).

8. Lum LC. Syndrome of chronic habitual hyperventilation. In: Hill O, ed. Modern trends in psychosomatic medicine. London: Butterworths, 1976:196-230.

9. Evans RW. Neurologic aspects of hyperventilation syndrome. Semin Neurol 1995;15:115-125.

10. Cohen B, Suzuki J-I. Eye movements induced by ampullary nerve stimulation. Am J Physiol 1963;204:347-351.

11. Minor LB, Tomko DL, Paige GD. Torsional eye movements evoked by unilateral labyrinthine galvanic polarizations in the squirrel monkey. In: Fetter M, Haslwanter T, Tweed D, eds. Three-dimensional kinematics of eye, head and limb movements. Amsterdam: Harwood Academic Publishers, 1997:161-170.

12. Fetter M, Sievering F. Three-dimensional eye movement anal- 
ysis in benign paroxysmal positioning vertigo and nystagmus. Acta Otolaryngol (Stockh) 1995;115:353-357.

13. Halmagyi GM, Curthoys IS, Cremer PD, et al. The human horizontal vestibulo-ocular reflex in response to highacceleration stimulation before and after unilateral vestibular neurectomy. Exp Brain Res 1990;81:479-490.

14. Straumann D, Zee DS, Solomon D, Lasker AG, Roberts DC. Transient torsion during and after saccades. Vis Res 1995;35: 3321-3334.

15. Holden JR, Wearne SL, Curthoys IS. A fast, portable desaccading program. J Vestib Res 1992;2:175-179.

16. Blanks RHI, Curthoys IS, Markham CH. Planar relationships of the semicircular canals in man. Acta Otolaryngol (Stockh) 1975;80:185-196.

17. Tweed D, Sievering D, Misslisch H, Fetter M, Zee D, Koenig E. Rotational kinematics of the human vestibuloocular reflex I. Gain matrices. J Neurophysiol 1994;72:2467-2479.

18. Proctor L, Dix R, Hughes D, Rentea R. Stimulation of the vestibular receptor by means of step temperature changes during continuous aural irrigation. Acta Otolaryngol (Stockh) 1975;79:425-435.

19. Jongkees LBW, Maas JPM, Philipszoon AJ. Clinical nystagmography. A detailed study of electronystagmography in 341 patients with vertigo. Pract Otorhinolaryngol (Basel) 1962;24: 65-93.

20. Tusa RJ, Grant MP, Buettner UW, Herdman SJ, Zee DS. The contribution of the vertical semicircular canals to highvelocity horizontal vestibulo-ocular reflex (VOR) in normal subjects and patients with unilateral vestibular nerve section. Acta Otolaryngol (Stockh) 1996;116:507-512.

21. Glasscock 3d ME, Hays JW, Minor LB, Haynes DS, Carrasco VN. Preservation of hearing in surgery for acoustic neuromas. J Neurosurg 1993;78:864-870.

22. Dornhoffer JL, Helms J, Hoehmann DH. Presentation and diagnosis of small acoustic tumors. Otalaryngol Head Neck Surg 1994;111:232-235.

23. Hirsch A, Anderson H. Audiologic test results in 96 patients with tumours affecting the eighth nerve. A clinical study with emphasis on the early audiological diagnosis. Acta Otolaryngol Suppl (Stockh) 1980;369:1-26.

24. Lustig LR, Rifkin S, Jackler RK, Pitts LH. Acoustic neuromas presenting with normal or symmetrical hearing: factors associated with diagnosis and outcome. Am J Otol 1998;19:212218.

25. Curtin HD. Rule out eighth nerve tumor: contrast-enhanced T1-weighted or high-resolution T2-weighted MR? AJNR Am J Neuroradiol 1997;18:1834-1838.

26. Kumon Y, Sakaki S, Ohue S, Ohta S, Kikuchi K, Miki H.
Usefulness of heavily T2-weighted magnetic resonance imaging in patients with cerebellopontine angle tumors. Neurosurgery $1998 ; 43: 1338-1343$.

27. Daniels RL, Shelton C, Harnsberger HR. Ultra high resolution nonenhanced fast spin echo magnetic resonance imaging: cost-effective screening for acoustic neuroma in patients with sudden sensorineural hearing loss. Otolaryngol Head Neck Surg 1998;119:364-369.

28. Rasmussen AT. Studies of the VIIIth cranial nerve of man. Laryngoscope 1940;50:67-83.

29. Davies HD, Carroll WM, Mastaglia FL. Effects of hyperventilation on pattern-reversal visual evoked potentials in patients with demyelination. J Neurol Neurosurg Psychiatry 1986;49; 1392-1396.

30. Davis FA, Becker FO, Michael JA, Sorensen E. Effect of intravenous sodium bicarbonate, disodium edetate ( $\mathrm{Na}_{2}$ EDTA), and hyperventilation on visual and oculomotor signs in multiple sclerosis. J Neurol Neurosurg Psychiatry 1970;33:723732.

31. Bednarik J, Novotny O. Value of hyperventilation in patternreversal visual evoked potentials. J Neurol Neurosurg Psychiatry 1989;52:1107-1109.

32. Misslisch H, Tweed D, Fetter M, Sievering D, Koenig E. Rotational kinematics of the human vestibuloocular reflex III. Listing's law. J Neurophysiol 1994;72:2490-2502.

33. Thurtell MJ, Black RA, Halmagyi GM, Curthoys IS, Aw ST. Vertical eye position-dependence of the human vestibuloocular reflex during passive and active yaw head rotations. J Neurophysiol 1999;81:2415-2428.

34. Goldberg JM, Fernández C. Physiology of peripheral neurons innervating semicircular canals of the squirrel monkey III. Variations among units in their discharge properties. J Neurophysiol 1971;34:676-684.

35. Leigh RJ, Robinson DA, Zee DS. A hypothetical explanation for periodic alternating nystagmus: instability in the optokinetic-vestibular system. Ann NY Acad Sci 1981;374: 619-635.

36. Robinson DA. The use of matrices in analyzing the threedimensional behavior of the vestibulo-ocular reflex. Biol Cybern 1982;46:53-66.

37. Yakushin S, Dai M, Suzuki J, Raphan T, Cohen B. Semicircular canal contributions to the three-dimensional vestibuloocular reflex: a model-based approach. J Neurophysiol 1995;74: 2722-2738.

38. Fetter M, Misslisch H, Sievering D, Tweed D. Effects of full field visual input on the three-dimensional properties of the human vestibuloocular reflex. J Vestib Res 1995;5:201-209. 